\title{
Rheological Percolation Behaviour and Fracture Properties of Nanocomposites of MWCNTs and a Highly Crosslinked Aerospace-grade Epoxy Resin System
}

DOI:

10.1016/j.compositesa.2017.11.012

\section{Document Version \\ Accepted author manuscript}

Link to publication record in Manchester Research Explorer

Citation for published version (APA):

Wilkinson, A., \& Liu, Y. (2018). Rheological Percolation Behaviour and Fracture Properties of Nanocomposites of MWCNTs and a Highly Crosslinked Aerospace-grade Epoxy Resin System. Composites Part A: Applied Science and Manufacturing, 105, 97-107. https://doi.org/10.1016/j.compositesa.2017.11.012

Published in:

Composites Part A: Applied Science and Manufacturing

\section{Citing this paper}

Please note that where the full-text provided on Manchester Research Explorer is the Author Accepted Manuscript or Proof version this may differ from the final Published version. If citing, it is advised that you check and use the publisher's definitive version.

\section{General rights}

Copyright and moral rights for the publications made accessible in the Research Explorer are retained by the authors and/or other copyright owners and it is a condition of accessing publications that users recognise and abide by the legal requirements associated with these rights.

\section{Takedown policy}

If you believe that this document breaches copyright please refer to the University of Manchester's Takedown Procedures [http://man.ac.uk/04Y6Bo] or contact uml.scholarlycommunications@manchester.ac.uk providing relevant details, so we can investigate your claim.

\section{OPEN ACCESS}




\title{
Rheological Percolation Behaviour and Fracture Properties of Nanocomposites of MWCNTs and a Highly Crosslinked Aerospace-grade Epoxy Resin System.
}

\author{
Yan Liu, Arthur Wilkinson * \\ North West Composites Centre, School of Materials, \\ University of Manchester, Manchester M13 9PL, UK.
}

*Corresponding author. Tel.: +44 (0)161 3065691. E-mail: Arthur.Wilkinson@manchester.ac.uk

\begin{abstract}
The effects of incorporating MWCNT in an aerospace-grade high functionality epoxy resin system are reported. Oscillatory-shear rheology showed as-received MWCNT (AR-MWCNT) to have a low degree of interaction with the resin, and consequently $\mathrm{G}^{\prime}$ values were low, whereas $\mathrm{G}^{\prime}$ values for functionalized MWCNTs (amino and oxidised) were higher due to increased resin-nanotube interactions. The three approaches used to obtain rheological percolation thresholds all showed values to increase in an order reflecting the improved MWCNT dispersion imparted by functionalisation. Analysis using colloidal dispersion theory fitted better with the experimental data than statistical percolation theory, and more closely mirrors the clustering of MWCNT agglomerates to form a percolated network. Fracture studies showed toughness decreased with AR-MWCNT addition, whereas for functionalised MWCNT addition toughness increased. For AR-MWCNT nanocomposites, nanotube debonding predominated and large weakly-bonded agglomerates were formed which reduced fracture toughness. In contrast, functionalised MWCNTs mainly exhibited fracture and pull-out and formed smaller agglomerates which toughened the matrix via crack-deflection and pinning.
\end{abstract}

\section{AR-AR-AR-AR-}

\section{KEY WORDS}

Carbon nanotubes and nanofibers; Polymer-matrix composites (PMCs); Fracture toughness; Rheological properties.

\section{INTRODUCTION}

Epoxy resins reinforced with multiwall carbon nanotubes (MWCNTs) are one of the most widely studied polymer nanocomposites. Upon addition of small quantities of nanotubes (often $\leq 1 \mathrm{wt} \%$ ), the resultant nanocomposites typically exhibit higher electrical conductivity [1] and improvements in mechanical properties such as Young's modulus [2], flexural strength, flexural modulus [3-5] and fracture toughness [6]. Most of the studies in the literature used low-functionality epoxy resins (typically diepoxide resins based on the diglycidylether of bis-phenol A, DGEBA [7, 8] ) with few reported studies on typical aerospace epoxy resins of high functionality, which have much higher glass transition temperatures $\left(\mathrm{T}_{\mathrm{g}}\right)$ resulting from their higher crosslink density. 
The viscoelastic properties of polymer nanocomposites are highly dependent on the dispersion state of the nanofillers and the degree of polymer-filler interaction [9]. Thus, the rheological behaviour of a MWCNT/epoxy resin nanocomposite is affected significantly by the addition level and any surface modification of the nanotubes. A higher concentration with good dispersion and distribution implies shorter distances between individual MWCNTs and stronger interconnects between them [10], leading to the formation of a continuous network of nanotubes. The critical concentration at which this change is observed is referred to as the percolation threshold (PT) [11] and in rheological studies is the concentration at which a mechanically effective network forms between the MWCNTs. Despite using the same grade of MWCNTs, Jan and Samuel [12] measured a rheological PT of $0.2 \mathrm{wt.} \%$ in a DGEBA epoxy resin system, whereas Charpategui and Markaide [13] reported a rheological PT value of only $0.13 \mathrm{wt} . \%$ in a similar epoxy resin system. Variations in reported PT values can often be ascribed to differences in dispersion and distribution as well as in the aspect ratios of the MWCNTs, either from the use of different grades of MWCNTs or size reductions due to processing. Jin and Dong [14] studied the effects of different surface modifications (amine, acid and plasmaoxidation) of MWCNTs on the rheological storage modulus $\left(\mathrm{G}^{\prime}\right)$ of MWCNT/DGEBA epoxy resin suspensions. $\mathrm{G}^{\prime}$ values of systems with functionalized MWCNTs were higher than that of the untreated nanotubes, ascribed to increased resin-nanotube interactions for the surface-modified MWCNTs.

The addition of MWCNTs to cured resins often has significant effects on the fracture toughness of nanocomposites [8]. For example [12], upon addition of $0.34 \mathrm{wt} . \%$ of amino-functionalized MWCNT to a DEGBF-based epoxy resin $\mathrm{K}_{\mathrm{IC}}$ was reported to increase by approximately $84 \%$, whereas for an equivalent addition of unmodified MWCNT the increase in $\mathrm{K}_{\mathrm{IC}}$ was only $26 \%$. Few studies on higher functionality epoxy resin systems have been reported, with only one [15] using the same tri-functional epoxy resin system used in the currrent study. This study [15] reported that the addition of $0.3 \mathrm{wt} \%$ SWCNT decreased the fracture toughness by $24 \%$ compared to the neat resin, which was ascribed to initial poor dispersion of nanotubes in the resin and their reagglomeration during the initial stage of the relatively high temperature curing cycle, in which the resin viscosity decreased significantly.

The main aim of this study was to improve understanding of the effects of MWCNT in an aerospace-grade epoxy resin system. Therefore, a tri-functional epoxy resin (triglycidyl-p-aminophenol, TGPAP) was used as the main resin and diaminodiphenylsulfone (DDS) as the hardener. TGPAP was diluted with a low molar mass, bi-functional resin (diglycidyl ether of bisphenol F, DGBPF) which has been shown [16] to significantly reduce the viscosity of TGPAP (by six-fold at $25^{\circ} \mathrm{C}$ ) but with only a relatively small drop in $\mathrm{T}_{\mathrm{g}}$ from 257 to $227^{\circ} \mathrm{C}$ at full cure. The main factors studied are the effects of MWCNT addition level and nanotube functionalization (as-received, acid-oxidised and amino-functionalized), on the rheological behaviour of resin-MWCNT suspensions which is fundamental to their processing and the fracture behaviour of cured nanocomposites.

\section{EXPERIMENTAL}

\section{Pre-processing and characterization of MWCNTs.}

As-received MWCNTs (AR-MWCNT) Nanocyl NC7000 and amino-functionalized MWCNTs (NH-MWCNT) Nanocyl NC3152) were obtained from Nanocyl S.A, Belgium. Oxidized MWCNTs were prepared using the following procedure using fresh reagents for each preparation: $400 \mathrm{mg}$ of AR-MWCNTs were exposed to mixed acids (volume ratio $\mathrm{V}_{\mathrm{H} 2 \mathrm{SO} 4}: \mathrm{V}_{\mathrm{HNO} 3}=75: 25$ ) for $1 \mathrm{hr}$ in a glass jar in an ultrasonic bath (Elmasonic $\mathrm{P} 70 \mathrm{H}$, $650 \mathrm{~W}$, room temperature), then mechanically stirred at $400 \mathrm{rpm}$ for $4 \mathrm{hr}$ at $40{ }^{\circ} \mathrm{C}$. The resultant oxidised 
MWCNT were then filtered and washed with distilled water until neutral $\mathrm{pH}$ was determined for at least one minute using indicator paper. A portion of the oxidised MWCNTs were base-washed (to give BW-MWCNT) with a $0.01 \mathrm{M} \mathrm{NaOH}$ solution until the filtrate changed from yellow/brown to colourless, followed by washing with $0.01 \mathrm{M} \mathrm{HCl}$ and distilled water until neutral $\mathrm{pH}$ [17]. Before preparing nanocomposites from AR-, BW- or NH-MWCNTs, $200 \mathrm{mg}$ of MWCNTs were dispersed into $200 \mathrm{ml}$ of acetone for $1 \mathrm{hr}$ using the Elmasonic 650 $\mathrm{W}$ ultrasonic bath, and the dispersion was then left overnight to partially evaporate the acetone. The almost dry MWCNTs were then ground using a pestle and mortar to further reduce the size of any agglomerates, with some residual acetone retained to inhibit fracture of the MWCNTs during grinding. After grinding, the MWCNTs were dried in an oven at $80{ }^{\circ} \mathrm{C}$ until constant weight.

For FTIR (Nicolet 5700, Thermo Electron) $2 \mathrm{mg}$ of MWCNTs were pressed with $100 \mathrm{mg} \mathrm{KBr}$ to form a disc (10 mm diameter and $0.5 \mathrm{~mm}$ thick). Absorbance spectra of three specimens of each type of MWCNT were taken over 500-4000 $\mathrm{cm}^{-1}$ at a resolution of $2 \mathrm{~cm}^{-1}$. Transmission electron microscopy, TEM, (JEOL 2000FX AEM) was used to study the morphology of the range of MWCNTs. TEM specimens were prepared as follows: $1 \mathrm{mg}$ of MWCNTs were dispersed into $100 \mathrm{ml}$ of acetone using an ultrasonic bath for $1 \mathrm{hr}$, then one drop of the mixture was placed on a copper mesh (Agar S162) and left to dry.

\section{Preparation of Nanocomposites.}

A mixture of a tri-functional epoxy resin, triglycidyl-p-aminophenol (TGPAP, Araldite ${ }^{\circledR}$ MY0500, Huntsman), of equivalent weight $110 \mathrm{~g} \mathrm{~mol}^{-1}$, with a bi-functional epoxy resin, diglycidyl ether of bisphenol F (DGBPF, DER. 354, Dow), of equivalent weight $171 \mathrm{~g} \mathrm{~mol}^{-1}$, at a weight ratio of 1:0.44 was used as the epoxy resin. The hardener 4, 4' - diaminodiphenylsulfone (DDS, Aradur 976-1, Huntsman) was added to the TGPAP/DGBPF resin mixture at a weight ratio of 1:0.25, giving a stoichiometric ratio of 1.0 for amine $[\mathrm{H}]$ :[Epoxide]. Three types of MWCNTs (AR-MWCNTs, BW-MWCNTs and NH-MWCNTs) were dispersed into epoxy resin mixes, initially using mechanical stirring (Heidolph RZR 2052) for $30 \mathrm{mins}$ at $2000 \mathrm{rpm}$ in an oil bath at $80{ }^{\circ} \mathrm{C}$. The resin-MWCNT dispersions were then subjected to three-roll milling (T65, Torrey Hills Technologies) for 15 minutes at room temperature at 50\% maximum rpm (feeder roll $54 \mathrm{rpm}$, centre roll $108 \mathrm{rpm}$, apron roll 216 $\mathrm{rpm})$. To form nanocomposites, the DDS hardener was added into the MWCNT-epoxy resin dispersion and mechanically stirred at $130{ }^{\circ} \mathrm{C}$ for 15 mins, this system was degassed in a vacuum oven at $100{ }^{\circ} \mathrm{C}$ for $30 \mathrm{~min}$ and then cast into a mould and cured at $130{ }^{\circ} \mathrm{C}$ for $1 \mathrm{hr}, 150{ }^{\circ} \mathrm{C}$ for $2 \mathrm{hr}$ and finally $200{ }^{\circ} \mathrm{C}$ for $2 \mathrm{hr}$.

\section{Rheology}

Rheological measurements were carried out at $80{ }^{\circ} \mathrm{C}$ using a Thermo Fisher MARS II Rheometer using $20 \mathrm{~mm}$ diameter parallel plates with a $0.5 \mathrm{~mm}$ gap. A frequency sweep was conducted on the liquid nanocomposite system (resins/MWCNTs without hardener) at a low controlled stress (1Pa) between 0.1 and $100 \mathrm{rad} / \mathrm{s}$, which is within the linear viscoelastic range (LVR), as determined by a stress amplitude sweep within the range 0.1 to $1000 \mathrm{~Pa}$ at a frequency of $1 \mathrm{~Hz}$.

\section{Dynamic Mechanical Thermal Analysis (DMTA)}

DMTA tests were conducted on a minimum of three specimens for each sample using a Perkin Elmer DMA 8000 in dual-cantilever bending mode. A $0.5 \mathrm{~mm}$ displacement amplitude was applied at a frequency of $1 \mathrm{~Hz}$ from $40{ }^{\circ} \mathrm{C}$ to $280{ }^{\circ} \mathrm{C}$ increasing at a rate of $5{ }^{\circ} \mathrm{C} / \mathrm{min}$. Matrix $\mathrm{T}_{\mathrm{g}}$ values were recorded as the peak of the $\tan \delta$ curve 


\section{Fracture Testing}

The critical stress-intensity factor (fracture toughness), $\mathrm{K}_{\mathrm{IC}}$, and the critical strain-energy release rate at fracture initiation (fracture energy), $\mathrm{G}_{\mathrm{IC}}$, of nanocomposites were measured using single edge-notch bending (SENB) in accordance with ASTM D5045. The tests were performed using an Instron 4411 fitted with a $500 \mathrm{~N}$ load cell at a crosshead speed of $10 \mathrm{~mm} / \mathrm{min}$ as recommended by the standard. At least 6 specimens of dimensions $44 \mathrm{x} 10$ x $5 \mathrm{~mm}$ were tested for each sample. The notch tip was machined using a precise cutting saw (ATM brilliant 221) and the crack was generated by tapping a fresh razor blade into the notch tip.

The fracture surfaces of nanocomposites after SENB testing were observed using scanning electron microscopy (SEM, Philips XL30 FEG). Specimens for SEM were cleaned using ethanol in an ultrasonic bath, stuck onto aluminium stubs with conductive carbon tape and then coated with a layer of Au/Pd alloy using a Gatan 682 Coating System.
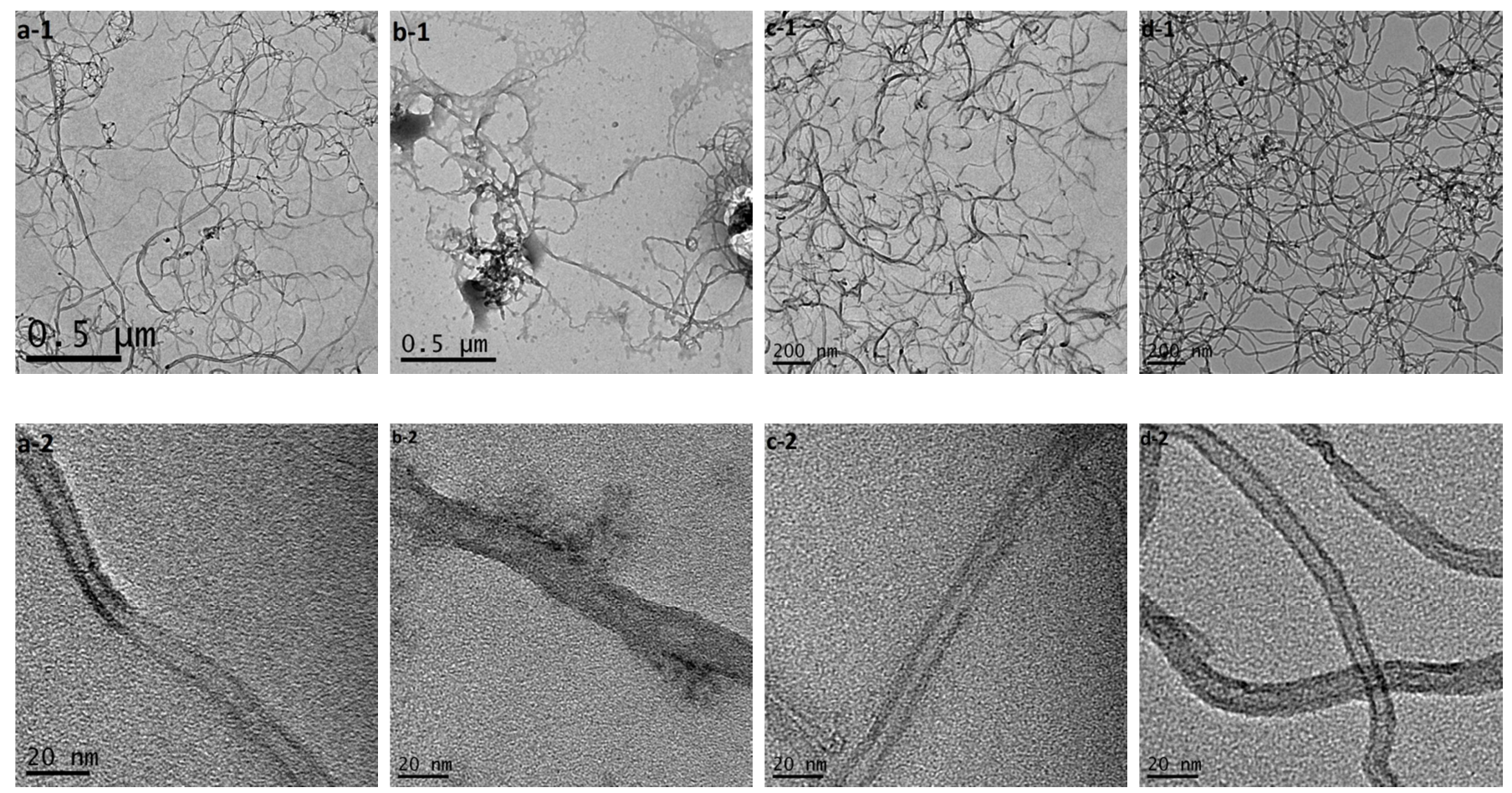

Figure 1. Typical TEM micrographs of the four types of MWCNTs at two magnifications $(1=6 \mathrm{k}, 2=$ 80k): a, AR-MWCNTs; b, oxidised-MWCNT; c, BW-MWCNT and d, NH-MWCNT.

\section{RESULTS AND DISCUSSION}

\section{Characterization of MWCNTs.}

Typical TEM micrographs of the AR-, oxidised-, BW- and NH-MWCNTs are shown in Figure 1. Compared to the other three types of MWCNTs, the oxidised-MWCNTs were more difficult to disperse in acetone and onto the grids due to the existence of the debris shown in Figure 1.b-1. The debris not only resides on the outer walls of the MWCNTs but also penetrates the MWCNT (Figure 1.b-2). Consequently, the oxidised-MWCNTs were not chosen as part of the epoxy nanocomposite study as the debris may affect their rheological and mechanical properties [17]. The average diameters of the MWCNTs were measured from the TEM micrographs using 
Image $\mathbf{J}$ image processing software $(\mathrm{NIH})$. The diameter of AR-MWCNTs $(6.89 \pm 1.16 \mathrm{~nm})$ reduced to 5.75 $( \pm 1.22) \mathrm{nm}$ following the acidification process, as measured from the BW-MWCNT as the diameters of oxidised-MWCNTs were hard to measure due to the debris. The average diameter of the NH-MWCNTs was measured at $9.5( \pm 1.81) \mathrm{nm}$, in agreement with the manufacturer's data.

In all the MWCNT FTIR spectra in Figure 2 (curves a-d) there are O-H stretching vibrations in the range 3600$3200 \mathrm{~cm}^{-1}$, due to hydroxyl groups and water; and the intensities of the O-H peaks in the spectra of BWMWCNT and oxidised-MWCNT are much stronger following oxidization in agreement with reported results [17-19]. The small peak at $1120 \mathrm{~cm}^{-1}$ in the spectrum of the NH-MWCNT (curve d) indicates the existence of a low concentration of $\mathrm{NH}_{2}$ groups, which is in accordance with the data provided by the supplier (weight percentage of $\mathrm{NH}_{2}$ group $<0.5$ wt. \%). In both the oxidised-MWCNT and BW-MWCNT curves, peaks at 1100 $\mathrm{cm}^{-1}$ and $1373 \mathrm{~cm}^{-1}$ indicating $\mathrm{C}-\mathrm{O}$ stretching and $\mathrm{O}-\mathrm{H}$ bending vibrations were detected. For oxidisedMWCNTs (curve b), the O-H peak is wider than that for the BW-MWCNTs (curve c) and peaks below 1000 $\mathrm{cm}^{-1}$ (e.g. $890 \mathrm{~cm}^{-1}$ and $600 \mathrm{~cm}^{-1}$ ) indicate $\mathrm{OH}-\mathrm{O}$ and $\mathrm{C}-\mathrm{C}=\mathrm{O}$ structures of $-\mathrm{COOH}$ groups.

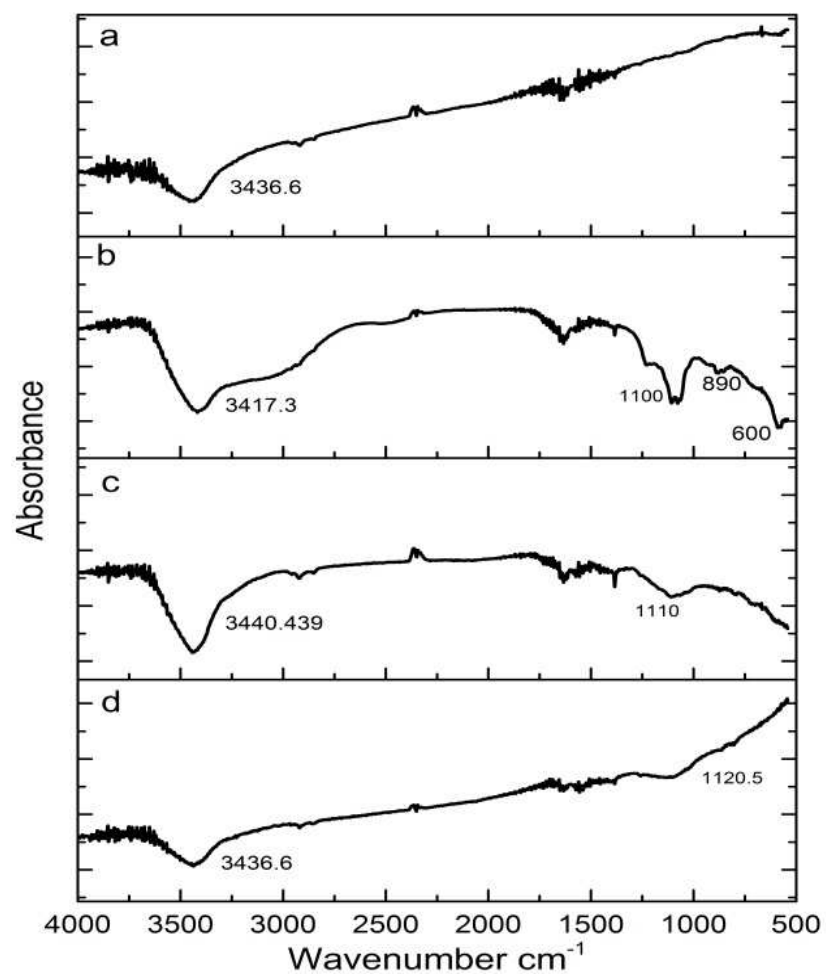

Figure 2 FTIR spectra of the MWCNTs (a, AR-MWCNT; b, oxidised-MWCNT; c, BW-MWCNT; d, NHMWCNT)

\section{Oscillatory-shear Rheology.}

Figure 3 shows the effects of MWCNT type and addition level on storage modulus $\left(\mathrm{G}^{\prime}\right)$ values. In general, $\mathrm{G}^{\prime}$ values of systems with functionalized MWCNTs were higher than those for AR-MWCNT, due to increased resin-nanotube interactions for the surface-modified MWCNTs in agreement with results for MWCNT/DGEBA resin systems [14], and $\mathrm{G}^{\prime}$ values are also seen to increase with increasing MWCNT level. The viscoelastic properties of resin-nanofiller dispersions in dynamic rheology measurements are sensitive to both the dispersion state of the filler and interaction between the filler and the resin $[9,20]$, particularly when the nanofillers begin 
to form a percolated network at a critical addition level, i.e. the percolation threshold (PT). CVD-grown MWCNT such as AR-MWCNT are highly entangled, and retain a degree of entanglement even after intensive shear mixing [4]. The lowest PTs reported for epoxy nanocomposites were for systems based on MWCNTs grown in aligned mats (which results in no entanglements) and where the formation of percolated networks is dominated by the reagglomeration of individual nanotubes. Surface-treated MWCNTs, such as the BW- and NH-MWCNTs used in this study tend to exhibit increased dispersion, or in other words a reduced degree of residual entanglement, resulting in lower percolation thresholds (PTs) than untreated MWCNTs. In this study, three approaches were used to study the rheological (PTs) of the epoxy-MWCNT dispersions: experimental observation, colloidal dispersion theory and statistical percolation theory.
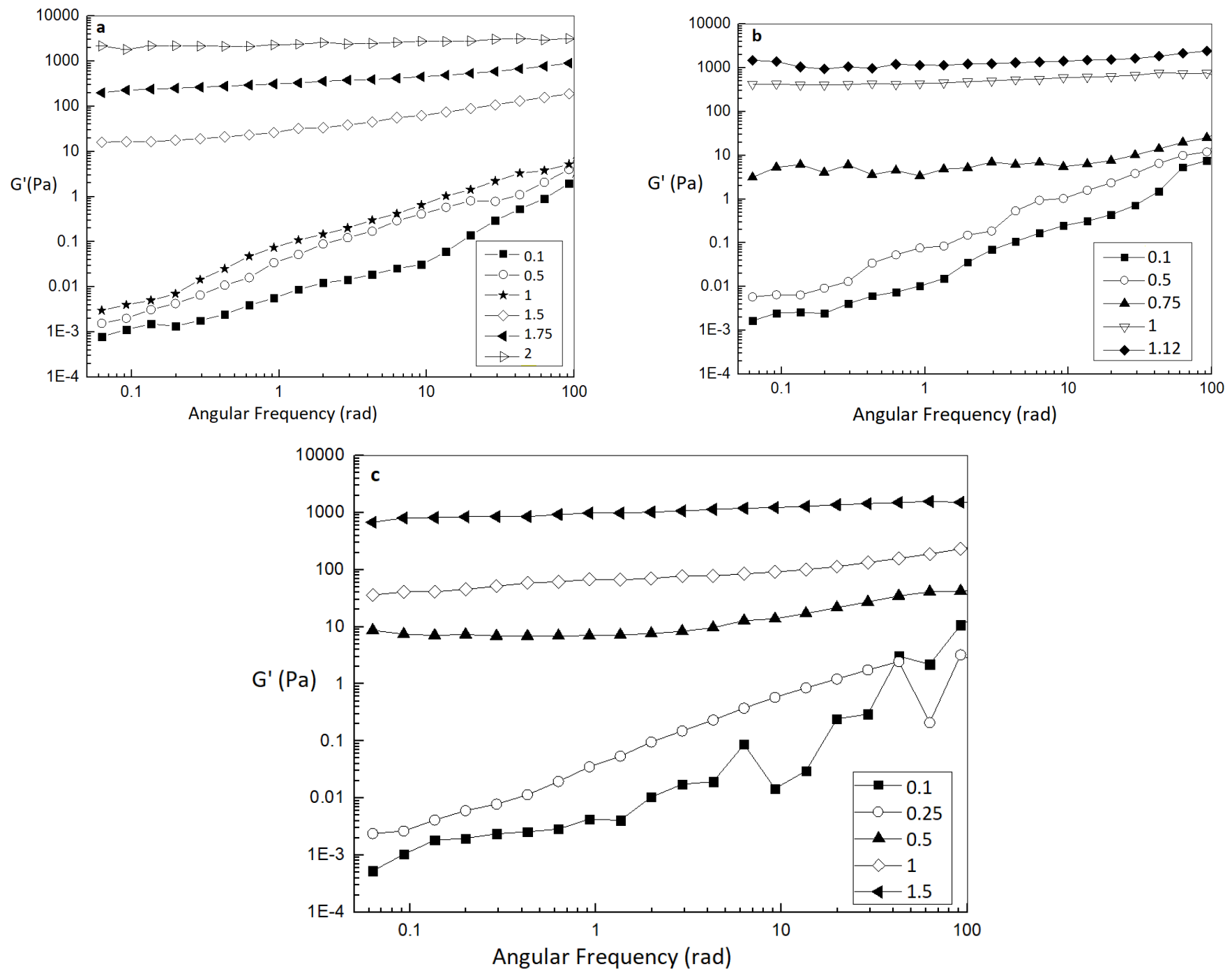

Figure 3 Shear storage modulus (G') vs. angular frequency data for MWCNTs-TGPAP/DEGBF dispersions (a, AR-MWCNT; b, BW-MWCNT; c, NH-MWCNT) showing the effects of MWCNT level.

\section{Approach I-Experimental Observation.}

In Figure $3 \mathrm{G}^{\prime}$ values increase with MWCNT level and eventually become independent of frequency, indicating the formation of an elastic network of MWCNTs at the PT [5,6]. This occurs in the AR-MWCNT system at levels of 1.75 and $2 \mathrm{wt} \%$, therefore the rheological percolation of this system $\left(p_{c, \text { exp }}\right)$ occurred in the region of 1.5-1.75 wt. \%. Similarly, $p_{c, \exp }(\mathrm{BW}-\mathrm{MWCNT})$ and $p_{c, \exp }(\mathrm{NH}-\mathrm{MWCNT})$ are observed at $0.75-1.0 \mathrm{wt} \%$ and 
0.25-0.5 wt. \%, respectively. The $p_{c, \exp }$ of the amine-functionalized NH-MWCNT system is lower than that of the AR-MWCNT system, which results from increased nanotube dispersion (in this case a reduced degree of residual entanglement following 3-roll milling) due to both reduced tube length (manufacturer's data for length is $<1 \mu \mathrm{m}$ compared to $1.5 \mu \mathrm{m}$ for the AR-MWCNTs) which will aid tubes escaping entanglements and reduced inter-tube interactions because of the functional groups on the surface of the NH-MWCNTs [21, 22].
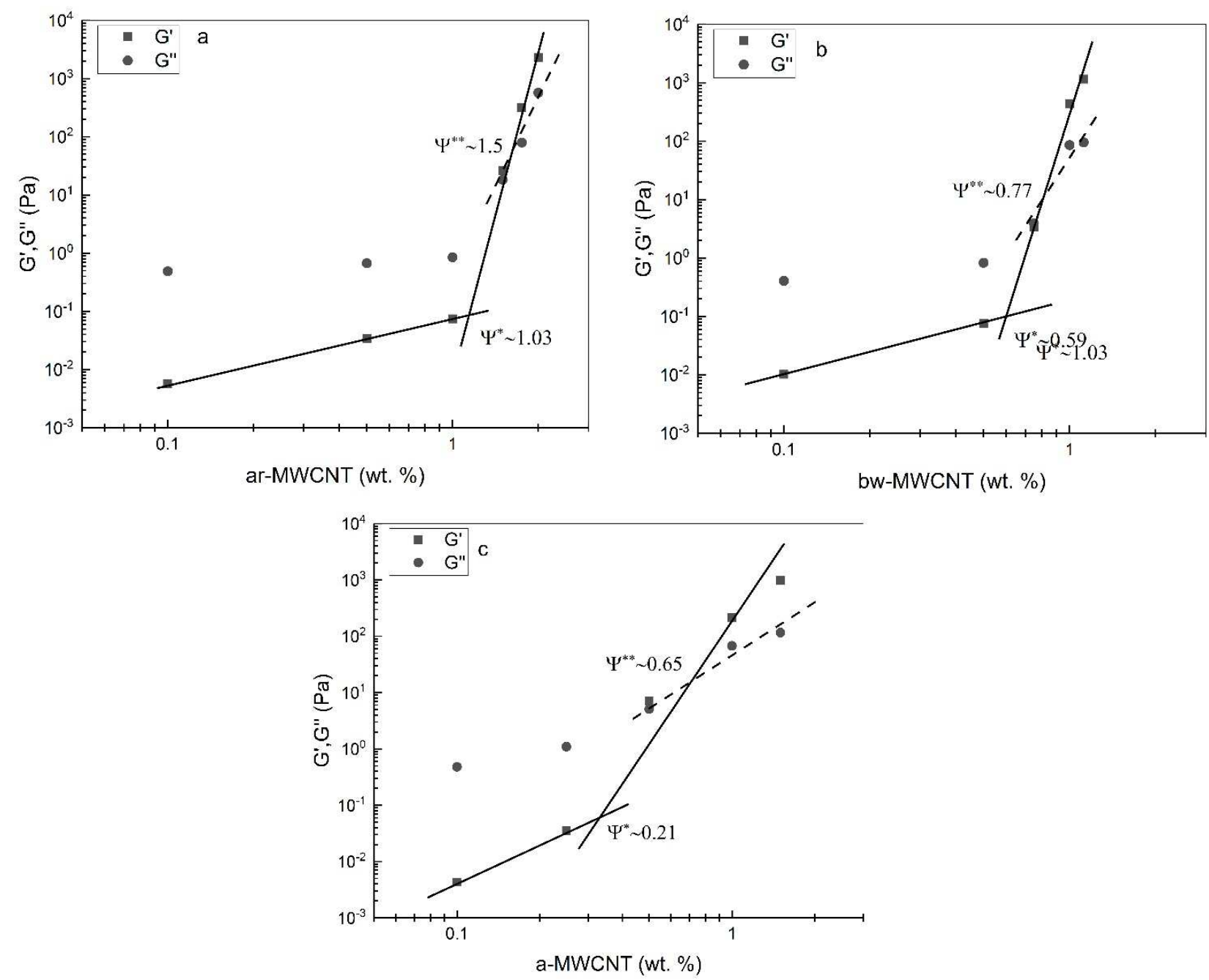

Figure 4 Plot of $G^{\prime}$ and $G^{\prime \prime}$ at a low frequency $(\omega=1 \mathrm{rad} / \mathrm{s}) \mathrm{vs.} \mathrm{MWCNT} \mathrm{loading} \mathrm{(a,} \mathrm{AR-MWCNT;} \mathrm{b,}$ BW-MWCNT; c, NH-MWCNT).

\section{Approach II--Colloidal Dispersion Theory.}

According to colloidal dispersion theory [21, 23, 24], agglomerates of colloidal particles will form with increasing filler content, which will then associate leading to clusters of agglomerates (fractal clusters) at relatively low filler levels and then finally to a percolated network at higher filler content. Thus, two PTs would be observed in such a nanocomposite system. The experimental data for $G^{\prime}$ and $G^{\prime \prime}$ (loss modulus) were fitted to the following power law expression:

$$
\boldsymbol{G}^{\prime} \sim \boldsymbol{\psi}^{\mu}
$$

Where $\mathrm{G}^{\prime}$ is the storage modulus at low frequencies at different MWCNT levels, $\psi$ is the MWCNT level and $\mu$ is a scaling exponent, expressing the strength of the filler structure. As shown in Figure 4, $\psi^{*}$ was determined 
by the inflexion point of the $\mathrm{G}^{\prime}$ data and depicts the formation of fractal clusters and $\psi^{* *}$ was obtained by the crossover at $\mathrm{G}^{\prime}=\mathrm{G}^{\prime \prime}$ and represents the formation of a continuous structural network composed of these clusters. When $\psi$ is $\left\langle\psi^{*}\right.$, a nanocomposite system behaves as a viscous liquid with $\mathrm{G}^{\prime}<\mathrm{G}^{\prime \prime}$, whereas when $\psi>\psi^{* *}$ the system shows a solid-like rheological response with $\mathrm{G}^{\prime}>\mathrm{G}^{\prime \prime}$.

Similar to the experimental observations (approach I), the $\psi^{*}$ and $\psi^{* *}$ PT values of both the functionalized MWCNT/epoxy resin systems are lower than those of the AR-MWCNT system. This implies that the lack of functional groups on AR-MWCNTs delayed the formation of clusters, probably as a result of relatively poor dispersion [11]. SEM studies of the cured nanocomposites, discussed later, showed that the agglomerates formed by the AR-MWCNTs are an order of magnitude larger than those formed by the functionalised MWCNTs. Thus, at AR-MWCNT addition levels $<1 \mathrm{wt} . \%$ the system probably contains relatively small numbers of large agglomerates which are less efficient at forming clusters. Zhu et al [21] used colloidal dispersion theory to calculate the rheological PTs of as-received CNF (carbon nano-fibre) and of silanized CNF in a DGBPF resin system. Their results showed similar behaviour to this study in that $\psi^{*}$ and $\psi^{* *}$ of the functionalized CNF system (at 0.3 wt. \% and 1.4 wt. \%) were lower than that of as-received CNF (0.5 wt. \% and 1.7 wt. \%).
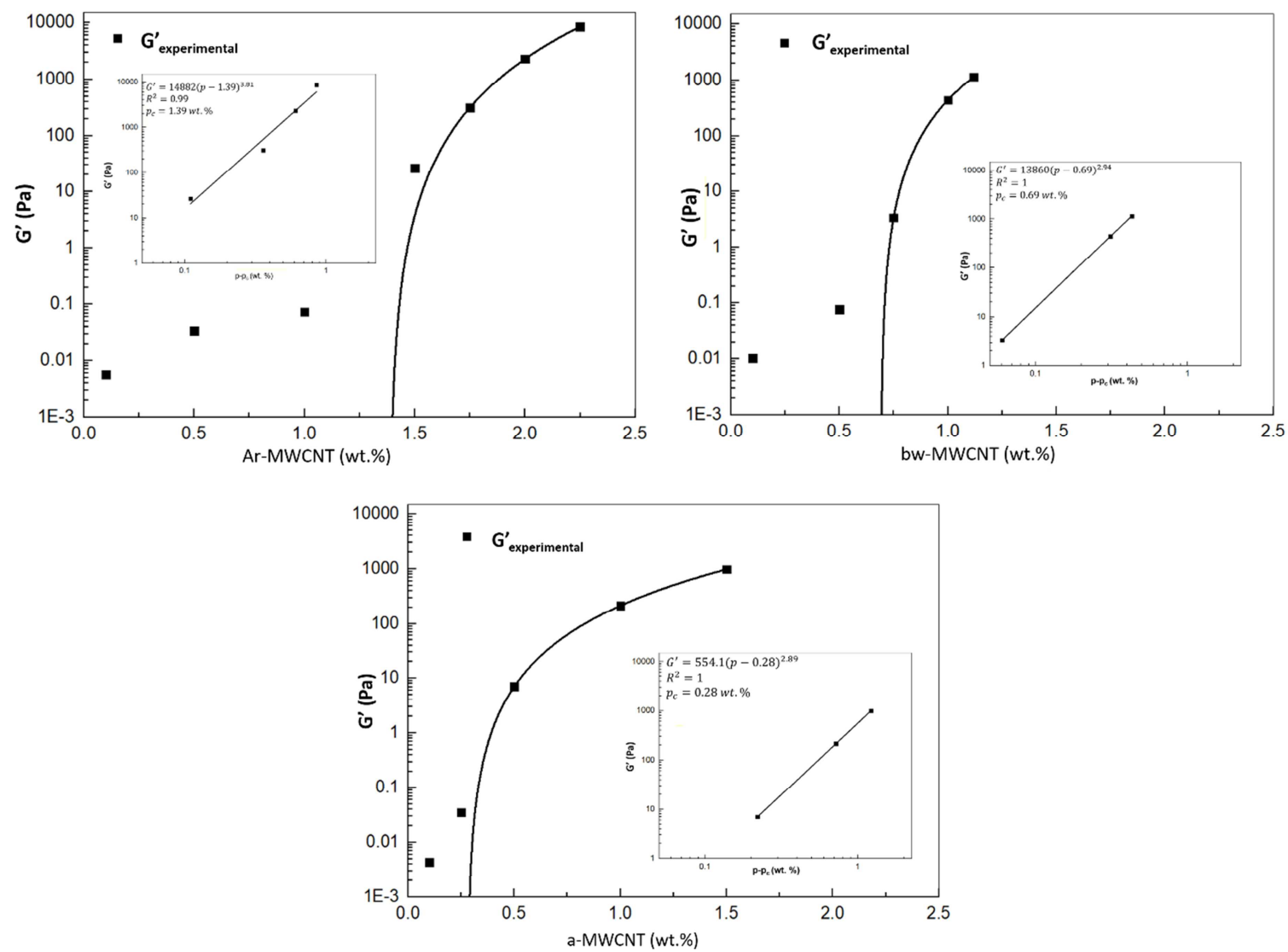

Figure 5 Rheological percolation thresholds determined using Equation 2. (a, AR-MWCNT; b, BWMWCNT; c, NH-MWCNT) 


\section{Approach III--Statistical Percolation Theory}

Statistical percolation theory, SPT [12, 25], which is widely used to determine the electrical PTs of nanocomposites, can also be used to obtain rheological PTs. Thus, by fitting the rheological data to equation 2 (Figure 5) the rheological PT is determined as the critical loading that initiates a rapid increase in $\mathrm{G}^{\prime}$.

$$
G^{\prime}=G_{0}\left(p-p_{c}\right)^{t}
$$

Where, $G_{0}$ is the proportionality constant, $p$ is the MWCNT loading, $p_{c}$ is the critical MWCNT loading and $t$ is the percolation exponential constant. Fitting the data gave $p_{c}$ values of $1.39,0.69$ and $0.28 \mathrm{wt}$. \%, and $t$ values of 3.61, 2.94 and 2.89 for the AR-, BW- and NH-MWCNT systems, respectively. The calculated $t$ values are within the range reported for space-filling networks of particles, i.e. between 2 and 4.5 [26].

Table 1 Rheological percolation thresholds (wt.\%) determined using approach I $\left(p_{c, \text { exp }}\right)$, approach II $\left(\psi^{*}\right.$ and $\left.\psi^{* *}\right)$ and approach III $\left(p_{c}\right)$

\begin{tabular}{ccccc}
\hline & $p_{c, \exp }$ & $\psi^{*}$ & $\psi^{* *}$ & $p_{c}$ \\
\hline AR-MWCNT & $1.5-1.75$ & 1.03 & 1.5 & 1.39 \\
BW-MWCNT & $0.75-1.0$ & 0.59 & 0.77 & 0.69 \\
NH-MWCNT & $0.25-0.5$ & 0.21 & 0.65 & 0.28 \\
\hline
\end{tabular}

The results from the three approaches used to obtain rheological PTs are summarized in Table 1. For each approach, the value of PT increases in the order NH- $<$ BW- $<$ AR-MWCNT, reflecting the improved dispersion imparted by functionalisation. The value of $\psi^{* *}$ obtained from colloidal dispersion theory is the closest to $p_{c \text {,exp }}$, as the formation of a continuous structural network of MWCNTs is much easier to detect from observation of experimental data. The PT, $p_{c}$, obtained from SPT lies between $\psi^{*}$ and $\psi^{* *}$, and is lower than $p_{c \text {,exp. SPT }}$ determines the rheological percolation from a geometrical and probabilistic concept [27], whereas colloidal dispersion theory quantifies the formation of a MWCNT network and provides more detailed information on the sudden changes of the properties of the liquid- and solid-state nanocomposites during the percolation transition [28]. The formation of a strong, percolated MWCNT network was accompanied by significant increases in viscosity of the resin systems which became too high for them to be degassed effectively. Thus, fracture testing was restricted to NCs with $\leq 1.0$ wt. $\%$ AR-MWCNTs and $\leq 0.5$ wt. $\%$ of BW-MWCNTs or NH-MWCNTs as the introduction of voids due to entrapped air would have affected the measurement of fracture properties. These types of nanocomposites are being assessed for use as matrices to produce multiscale laminate composites with carbon fibre fabrics by resin film infusion [29]. The films used in this technique may contain different levels of MWCNTs; with relatively low levels (i.e. < PT) used for films aimed at providing interlaminar toughening and higher levels $(>$ PT) used for films aimed at providing functionality (such as electrical conductivity) that requires the formation of a percolated network.

\section{DMTA}

Figure 6 shows plots of DMTA data vs, temperature for the unfilled epoxy resin matrix and nanocomposites with different types of MWCNTs at $0.1 \mathrm{wt}$. $\%$, and values derived from these plots are given in Table 2 . The addition of 0.1 wt. \% AR-MWCNTs had little effect on both $\mathrm{E}^{\prime}$ and $\mathrm{T}_{\mathrm{g}}$. This contrasts with the effects of the functionalized MWCNTs, for which compared to the unfilled resin $\mathrm{E}^{\prime}$ at $100{ }^{\circ} \mathrm{C}$ increased by $22 \%$ and $48 \%$ on addition of BW-BW- and NH-MWCNTs, respectively, indicative of improved dispersion and stronger interfacial interaction with the matrix. However, the $\mathrm{T}_{\mathrm{g}}$ values of the nanocomposites changed little compared 
to the unfilled resin, with NH-MWCNTs giving the greatest shift of $+3{ }^{\circ} \mathrm{C}$. Similar results, with significant improvements in $\mathrm{E}^{\prime}$ but small increases in $\mathrm{T}_{\mathrm{g}}$, have been reported for other epoxy-CNT systems [30].

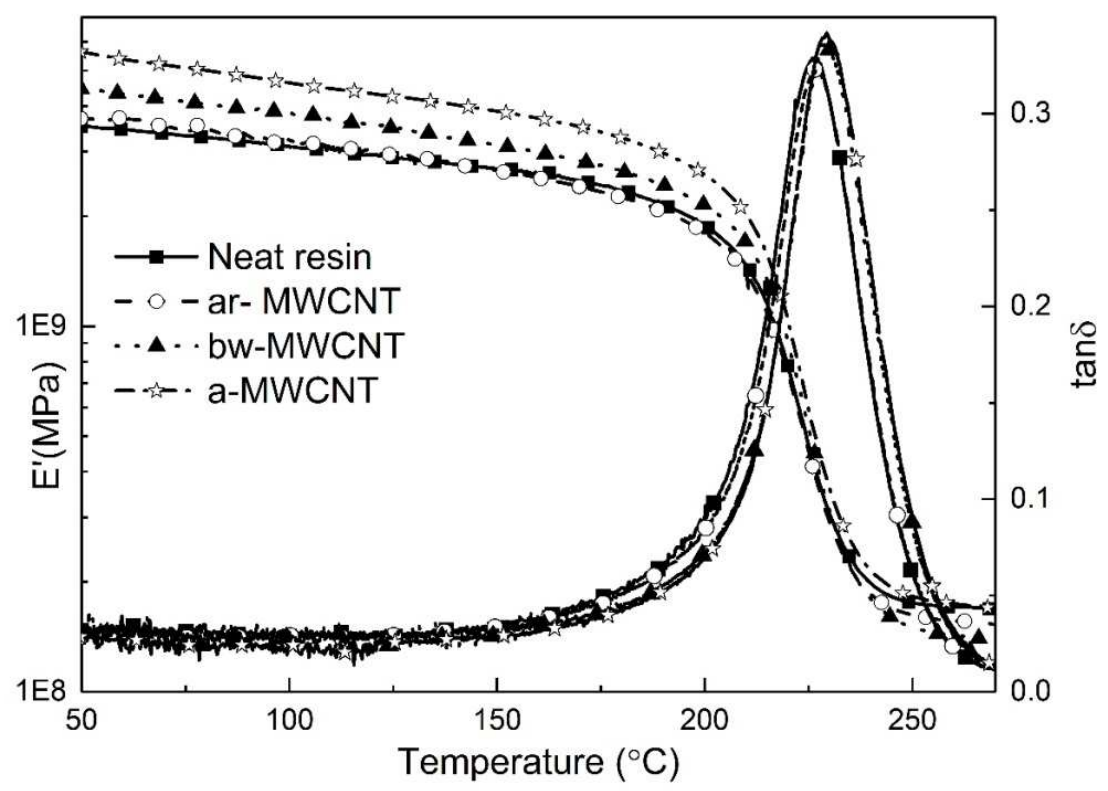

Figure 6 Storage modulus, $E^{\prime}$, and loss factor, tan $\delta$, vs. temperature for cured nanocomposites with MWCNTs at 0.1 wt. \%.

Table 2 DMTA data for nanocomposites with MWCNTs at $0.1 \mathrm{wt} . \%$..

\begin{tabular}{cccc}
\hline & $\mathrm{T}_{\mathrm{g}} /{ }^{\circ} \mathrm{C}$ & $\mathrm{E}^{\prime}$ at $100{ }^{\circ} \mathrm{C} / \mathrm{GPa}$ & $\tan \delta$ \\
\hline Unfilled resin & $226.6 \pm 0.8$ & $3.1 \pm 1.0$ & $0.33 \pm 0.01$ \\
AR-MWCNT & $226.5 \pm 0.8$ & $3.3 \pm 0.2$ & $0.32 \pm 0.01$ \\
BW-BW-MWCNT & $228.8 \pm 0.4$ & $3.8 \pm 0.4$ & $0.34 \pm 0.01$ \\
NH-MWCNT & $229.4 \pm 0.5$ & $4.6 \pm 0.5$ & $0.34 \pm 0.0$ \\
\hline
\end{tabular}

\section{Fracture Behaviour}

Figure 7 The critical stress intensity factor, KIC (a), and the critical strain energy release rate, GIC (b), of nanocomposites with different types of MWCNTs at levels up to $1 \mathrm{wt} . \%$. The fracture toughness of the unfilled resin is $0.68 \pm 0.04 \mathrm{MN} \cdot \mathrm{m}^{-1.5}$ which is lower than the $1.1 \pm 0.34$ reported previously for this system [31] and may be attributed to differences in stoichiometric ratio and curing conditions. In this study the stoichiometric ratio of 1.0 for amine $[\mathrm{H}]$ :[Epoxide] results in both primary and secondary amine groups reacting with the epoxide groups and leads to a high crosslink density, causing the toughness to decrease because of the formation of a less mobile network. The measured $\mathrm{K}_{\mathrm{IC}}$ in figure 7 is, however, equivalent to the value of $0.6 \pm 0.05$ measured for this system under equivalent formulation and curing conditions [32]. On addition of up to 0.5 wt. \% ARMWCNT, $K_{I C}$ is seen to decrease to $0.53 \pm 0.03$ but then increases to $0.61 \pm 0.04$ at 1.0 wt. \%. The functionalized MWCNT systems both show an initial drop on addition of $0.1 \mathrm{wt} \%$, but $\mathrm{K}_{\mathrm{IC}}$ then begins to recover and the highest $\mathrm{K}_{\mathrm{IC}}$ value of $0.71 \pm 0.05$ was measured for the $0.5 \mathrm{wt}$. \% NH-MWCNT system. However, this is only $4.4 \%$ higher than the unfilled resin. These results are in general agreement with Ashrafi et.al [31], who reported that addition of SWCNTs to a highly-crosslinked TGPAP/DDS system decreased $\mathrm{K}_{\mathrm{IC}}$ by up to $28 \%$. However, these results are in contrast to previous studies [8,33-35], albeit with very different resin systems, where $\mathrm{K}_{\mathrm{IC}}$ increased significantly with the addition level of as-received MWCNT in epoxy resin 
nanocomposites. Thus, the reduction in $\mathrm{K}_{\mathrm{IC}}$ may be related to the structure of the hardener and resins used as well as the high crosslink density [36] and to the high viscosity of aerospace-grade epoxy systems which may adversely affect the dispersion of MWCNTs [37]. In contrast to the AR-MWCNTs, the functional groups (especially $-\mathrm{NH}_{2}$ groups) on the surface of the functionalized MWCNTs have been proposed to reduce the degree of crosslinking at epoxy-MWCNT interfaces [38] producing a looser, more mobile network with increased fracture toughness [8].
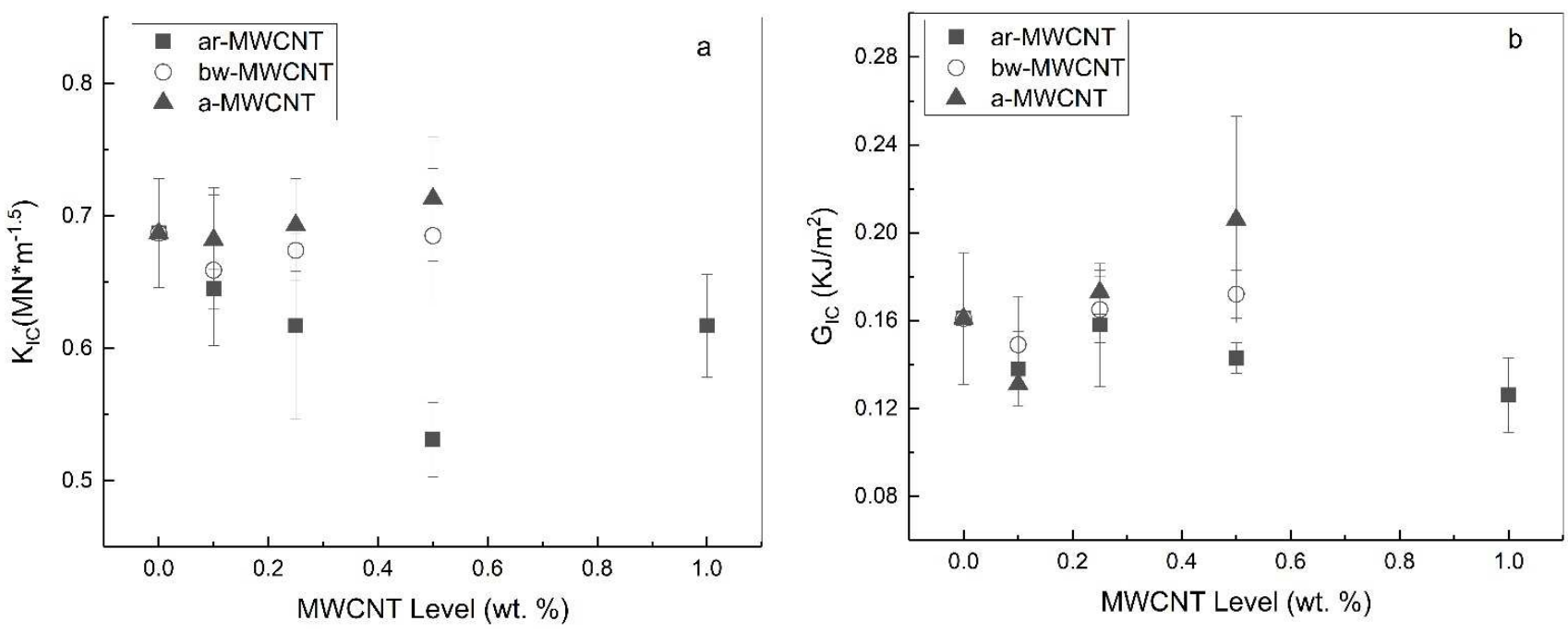

Figure 7 The critical stress intensity factor, $\mathrm{K}_{\mathrm{IC}}(\mathbf{a})$, and the critical strain energy release rate, $\mathrm{G}_{\mathrm{IC}}(\mathrm{b})$, of nanocomposites with different types of MWCNTs at levels up to 1 wt.\%.

Although a number of studies have reported on the fracture toughness testing of epoxy resin nanocomposites, such as [20-23, 31], values of fracture energy, $\mathrm{G}_{\mathrm{IC}}$, measured using this test method are very rarely reported. The measured $\mathrm{G}_{\mathrm{IC}}$ data are shown in Figure.$b$, and values for nanocomposites with functionalized MWCNTs are seen to increase along with MWCNT levels, whereas GIC $_{I C}$ decreases in the AR-MWCNT nanocomposites. The highest $\mathrm{G}_{\mathrm{IC}}$ values were measured for the $0.5 \mathrm{wt}$. $\%$ nanocomposites at $7 \%$ and $28 \%$ above the unfilled resin for the BW-MWCNT and NH-MWCNT systems, respectively. However, the addition of AR-MWCNT decreased $\mathrm{G}_{\mathrm{IC}}$ by $22 \%$ at the level of $1 \mathrm{wt}$. \%. Similar to the $\mathrm{K}_{\mathrm{IC}}$ results, the highest value of $\mathrm{G}_{\mathrm{IC}}$ was measured for the $0.5 \mathrm{wt} . \% \mathrm{NH}-\mathrm{MWCNT}$ at $206.3 \pm 47 \mathrm{~J} \cdot \mathrm{m}^{-2}$, which indicates that functional groups on the surface of MWCNTs may have loosened the network structure at the epoxy-MWCNT interface [25] [39] and increased energy absorbance in the nanocomposites.

\section{Toughening Mechanisms}

\section{Effects of MWCNT functionalization}

Figure shows typical fracture surfaces of the different types of MWCNT nanocomposites. In the AR-MWCNT nanocomposites cavities are observed (Figure a a-1) left by single MWCNTs as well as MWCNTs exposed along their length (Figure . a-2), behaviour which is unlike the MWCNT pull-out reported typically for DGEBA resin systems $[8,40]$. This failure mode is illustrated in Figure .d-1 and results from the weak interfacial bonding between the AR-MWCNT and the epoxy matrix. Debonding occurs along the tube length and the energy dissipation is much smaller than that of MWCNT pull-out and fracture (Figure .d-2) in which energy is absorbed due to friction between a nanotube and the matrix as pull-out occurs and by fracture of the nanotube 
(as the nanotubes are long, and not straight, they will tend to rupture rather than pull out completely).. In contrast, for BW-MWCNT nanocomposites (Figure .b) MWCNT fracture without pull-out (Figure .d-3) is the major failure mode along with a very small amount of MWCNT pull-out (highlighted by the white arrows). This is reflective of stronger interfacial bonding for BW- compared to AR-MWCNT, but the lack of pull-out may be indicative of tube damage caused during acidification. The behaviour of NH-MWCNT nanocomposites (Figure .c) is dominated by pull-out (white arrows) and tube fracture in which the nanotubes first debond from the matrix then pull-out (Figure .d-2). These different failure modes for functionalized MWCNT result in both $\mathrm{K}_{\mathrm{IC}}$ and $\mathrm{G}_{\mathrm{IC}}$ increasing for nanocomposites with higher MWCNT levels. Comparing the two functionalised MWCNT systems, the superior performance of NH-MWCNTs can be ascribed to covalent bonding between $\mathrm{NH}_{2}$ groups on the surface of NH-MWCNTs and the epoxy matrix which, as well as increasing interfacial bonding, have been proposed to reduce the degree of crosslinking at epoxy-MWCNT interfaces [38]. In addition, the predominance of tube fracture before pull-out in the BW-MWCNT nanocomposites may be indicative of damage caused during acidification reducing the strength of the nanotubes. The lack of pull out is a significant loss, as Hsieh at al [40] modelled the energy absorbtion due to pull-out of MWCNTs from an epoxy matrix to be at least equivalent to that resulting from fracture of the nanotubes.
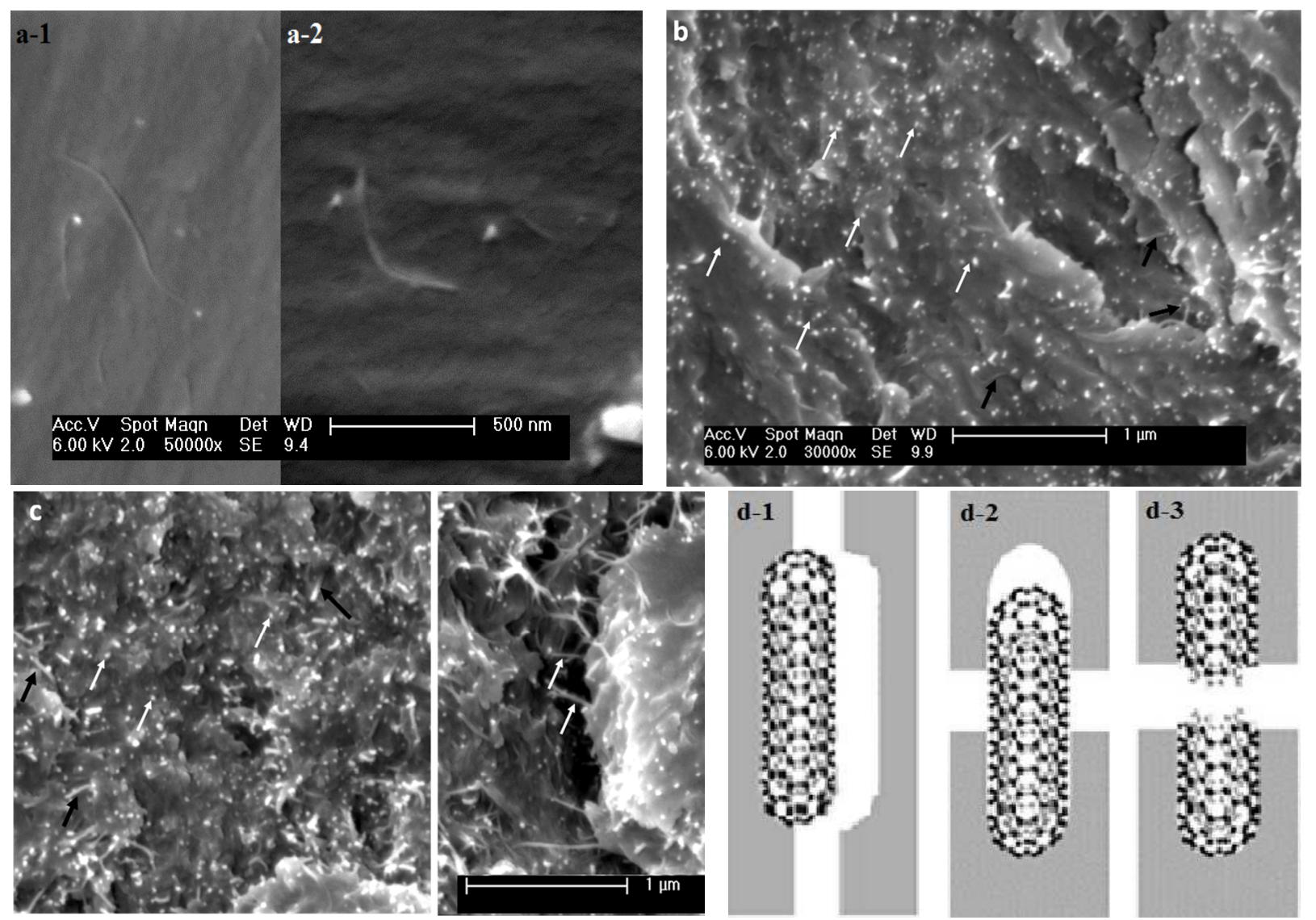

Figure 8 SEM micrographs of SENB fracture surface of nanocomposites with (a) AR-MWCNTs, (b) BWMWCNT, (c) NH-MWCNTs. (d) illustration of MWCNTs pull-out and fracture behaviour [34] 


\section{MWCNT Agglomeration}
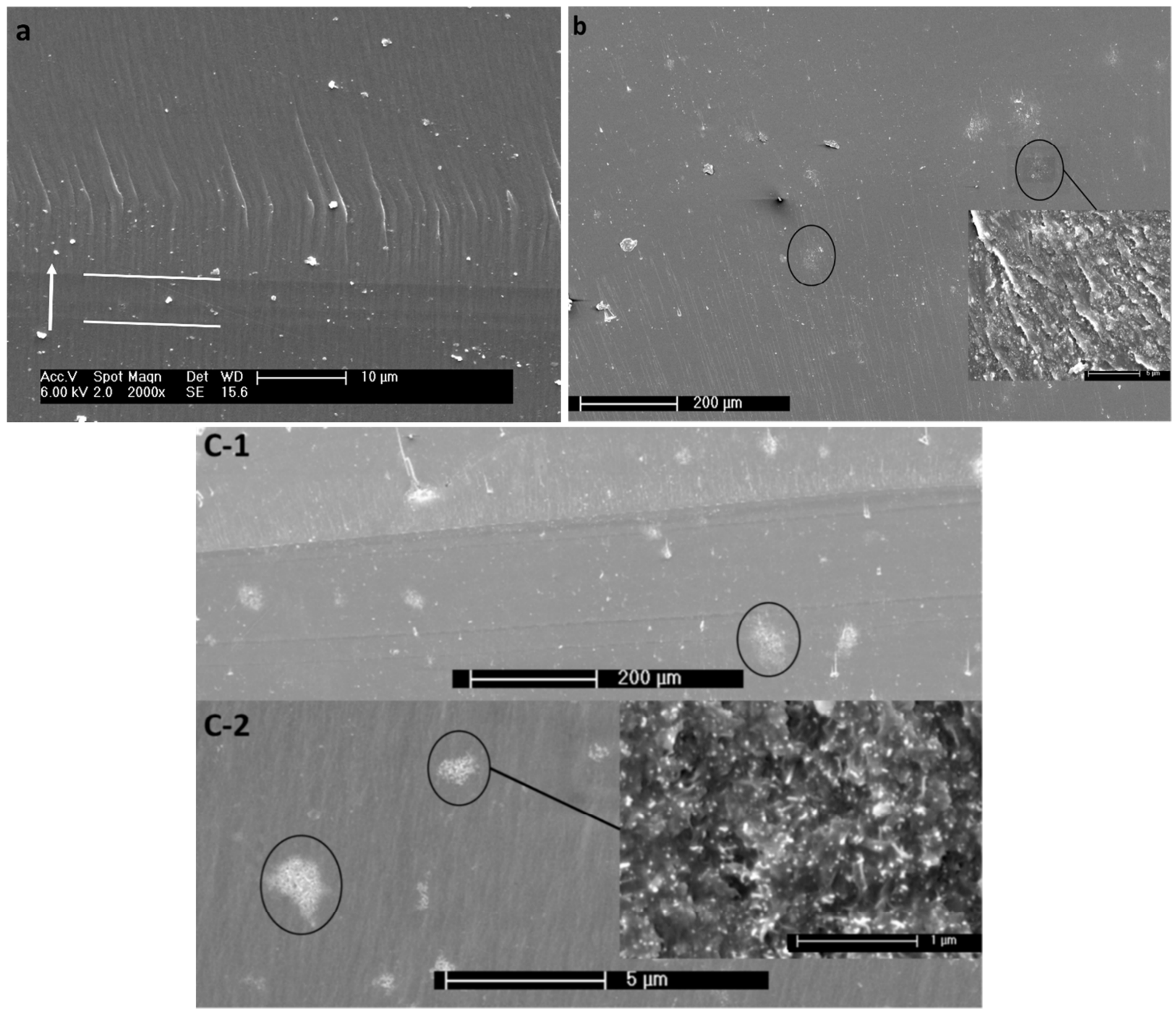

Figure 9 SEM micrographs of SENB fracture surfaces of AR-MWCNT/TGPAP-DGBPF nanocomposites with typical agglomerates circled. (a), unfilled resin; (b), 0.1 wt.\% AR-MWCNT; (c-1), 0.5 wt.\% ARMWCNTs; (c-2), 0.5 wt. \% NH-MWCNTs.

Figure .a shows a typical SEM micrograph of a fracture surface for the unfilled epoxy resin where the direction of crack propagation is indicated by the white arrow. Crack propagation takes place by a stick/slip mode [41], and crack arrest lines (white lines) are seen on the fracture surface between which the fracture surface is relatively smooth, characteristic of brittle fracture behaviour. The fracture surface of a nanocomposite with 0.1 wt. \% AR-MWCNTs (Figure .b) shows a smooth fracture surface similar to the unfilled resin but with a number of MWCNT agglomerates (identified by the black circles). However, fracture surfaces of nanocomposites with 0.5 wt. \% of AR-MWCNTs (Figure .c-1) and of NH-MWCNTs (Figure .c-2) show many more agglomerates. This is not unexpected; for example in [40] addition of 0.1 or $0.2 \mathrm{wt} . \%$ of MWCNTs resulted in pull-out of $100 \%$ of the nanotubes, whereas only $62 \%$ were observed to pull-out on addition of $0.5 \mathrm{wt} . \%$ due to increased nanotube agglomeration. The agglomerate sizes in the NH-MWCNT nanocomposites (typically $\leq 10 \mu \mathrm{m}$ in diameter) are also much smaller than those in the AR-MWCNT nanocomposites (typically 10 to $100 \mu \mathrm{m}$ ). This 
indicates that the fracture toughness may be affected by the distribution and size of the agglomerates, as $\mathrm{K}_{\mathrm{IC}}$ appears to increase along with the increased number of smaller agglomerates in functionalized MWCNT nanocomposites.

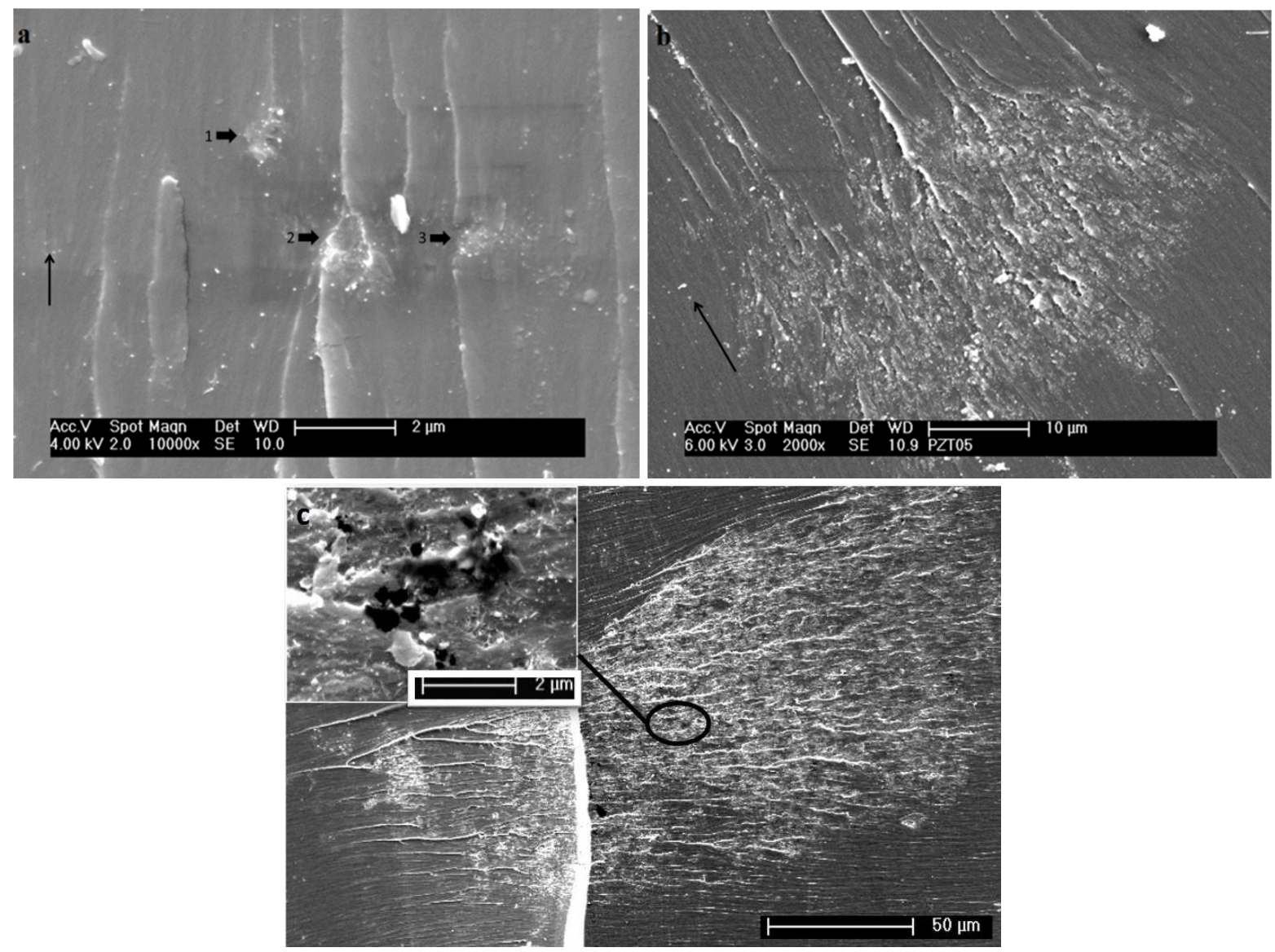

Figure 10 SEM micrographs of typical MWCNT agglomerates on the fracture surfaces of nanocomposites with 0.5 wt. \% AR-MWCNTs. The crack direction is shown by long arrows. (a) small agglomerates $(<10 \mu \mathrm{m}$ in diameter), showing crack-agglomerate interactions (indicated by arrows 1-3); (b) medium sized agglomerate $(10-100 \mu \mathrm{m}) ;(c)$ large agglomerate $(>100 \mu \mathrm{m})$. 


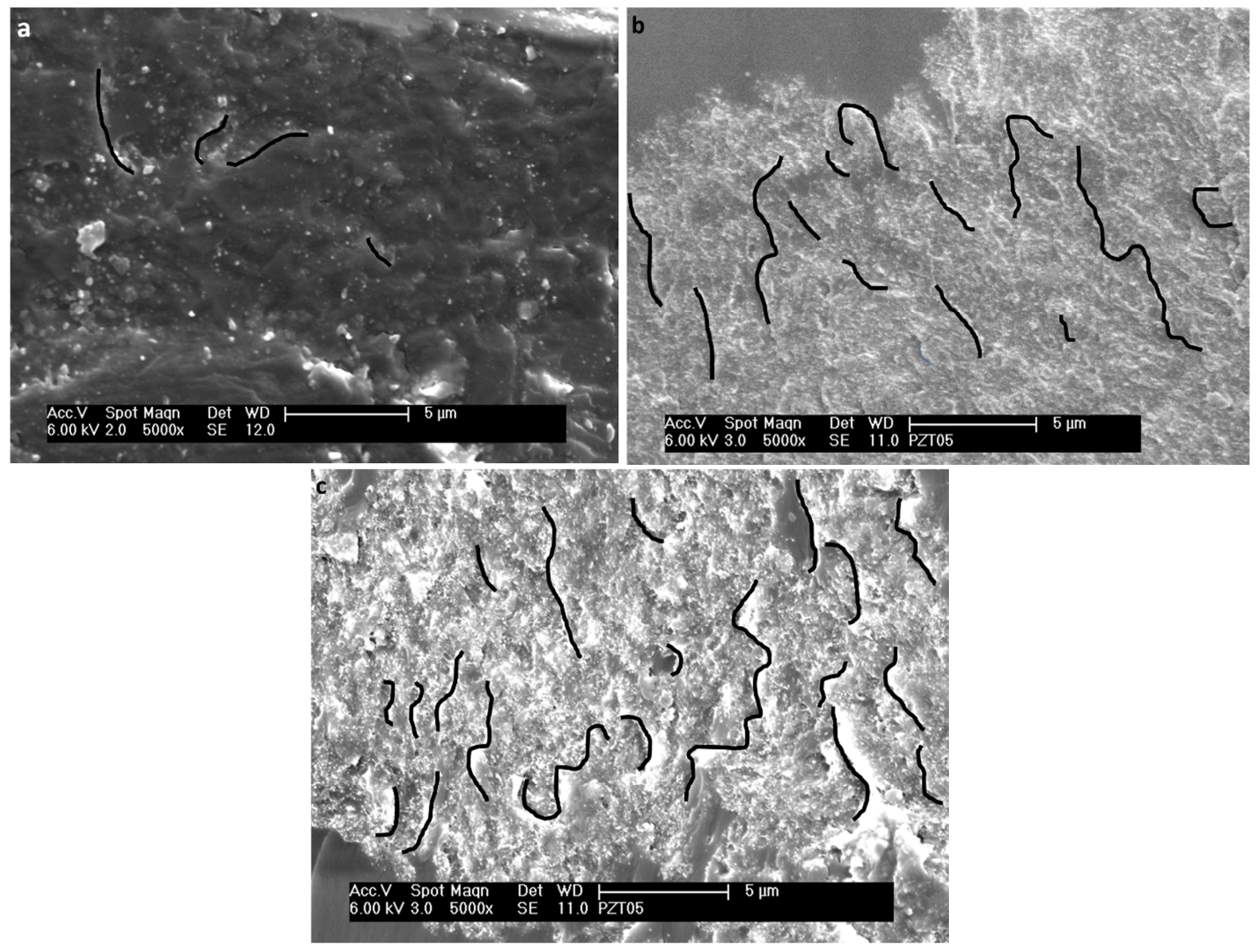

Figure 11 SEM micrographs of SENB fracture surface of medium-sized agglomerates in nanocomposites with different types of MWCNT all at 0.5 wt. \%. Ridge outlines are marked by black lines. (a, ARMWCNT; b, BW-MWCNT; c, NH-MWCNT)

The nanocomposite morphology observed from SEM studies is of an epoxy matrix containing dispersed MWCNTs, although the distribution of these dispersed nanotubes is not homogeneous, and MWCNT agglomerates of a range of sizes. The mixtures of MWCNT in epoxy resin appeared well dispersed and stable at room temperature; therefore it is possible that agglomerates formed due to reagglomeration of the MWCNT during the high temperature curing cycle of the nanocomposite, as observed by Ma et al [29]. The viscosity of the epoxy resin system used in this study has been measured to drop by an order of magnitude over the temperature range $40-130{ }^{\circ} \mathrm{C}$ [32]. This drop in viscosity will promote reagglomeration of the MWCNT, which will be resisted by the higher resin-tube interactions of the functionalised MWCNT. In this study, the sizes of the MWCNT agglomerates were classified into three groups: small ( $<10 \mu \mathrm{m}$, Figure 10.a.), mediumsized $(10-100 \mu \mathrm{m}$, Figure 10.b) and large (>100 $\mu \mathrm{m}$, Figure 10.c). Figure 10.a shows interactions between the agglomerates and cracks indicative of possible crack pinning and deflection, suggesting that the small agglomerates are behaving as rigid particles in their interactions with the advancing crack. Crack pinning is identified by the presence of bow lines on the fracture surface, as indicated by arrow 2 in Figure 10a, and typically tends to occur when the particle diameters are much larger than the crack-opening displacement $(\delta)$ which, under plane-strain conditions, can be calculated from the ratio of the fracture energy to the yield stress of the matrix $\left(\mathrm{G}_{\mathrm{IC}} / \sigma_{\mathrm{y}}\right)$ [42]. Using a value of $\sigma_{\mathrm{y}}$ for a TGPAP/DDS system of $150 \mathrm{MPa}$ [43] the values of $\delta$ calculated for the nanocomposites range from to 0.7 to $1.4 \mu \mathrm{m}$, thus crack pinning by the MWCNT 
agglomerates is a viable toughening micromechanism. Crack deflection occurs as the crack front encounters a particle and tilts and twists causing an increase in the fracture surface area. The micrographs in Figure 10 show significant increases in surface roughness as the crack encounters agglomerates indicating that crack deflection by the agglomerates is a viable toughening micromechanism. The smaller agglomerates that form in the nanocomposites with functionalised MWCNTs are greater in number (at equivalent wt.\%) and have a stronger MWCNT-matrix interface and therefore will act as a more rigid obstruction, resulting in more effective crack deflection. This is exemplified in Figure which compares fracture surfaces of medium-sized agglomerates for nanocomposites with 0.5 wt. $\%$ of the three MWCNT types. All The nanocomposites show ridges resulting from deflection of the crack plane, however the nanocomposites with functionalized MWCNTs (Figure .b and Figure .c) show many more and higher ridges than the nanocomposites with AR-MWCNTs (Figure .a) indicating more extensive crack deflection. There is also a significant difference between the nanocomposites with BW- and NH-MWCNTs (Figure .b and Figure .c, respectively) with the increased number of ridges on the fracture surface of the NH-MWCNT nanocomposite reflecting greater energy absorbtion; due to the occurrence of pull-out for the nanotubes in addition to fracture and to the rigid agglomerates containing more strongly bonded MWCNTs.

\section{CONCLUSIONS}

Oscillatory-shear rheology studies of AR-MWCNT, BW-MWCNT and NH-MWCNT systems showed G' values of systems with functionalized MWCNTs were higher than those for AR-MWCNT, due to the increased resin-nanotube interactions for the surface-modified MWCNTs. All three of the approaches used to obtain rheological PTs showed values to increase in the order NH- $<$ BW- $<$ AR-MWCNT, reflecting the improved dispersion imparted by functionalisation. Percolation analysis using colloidal dispersion theory fitted better with the experimental data than statistical percolation theory and the premise on which it is based more closely mirrors the behaviour of the MWCNTs in this high functionality epoxy system, namely the clustering of MWCNT agglomerates to form a percolated network.

SENB fracture studies showed that for AR-MWCNT nanocomposites both $\mathrm{K}_{\mathrm{IC}}$ and $\mathrm{G}_{\mathrm{IC}}$ decreased with MWCNT levels. While for MWCNT with functional groups, both $\mathrm{K}_{\mathrm{IC}}$ and $\mathrm{G}_{\mathrm{IC}}$ generally increased with MWCNT levels. The highest value of $\mathrm{K}_{\mathrm{IC}}\left(0.713 \mathrm{MN} \cdot \mathrm{m}^{1.5}\right)$ and $\mathrm{G}_{\mathrm{IC}}\left(206.3 \mathrm{~J} \mathrm{~m}^{-2}\right)$ were obtained from the nanocomposite with $0.5 \mathrm{wt}$. \% NH-MWCNTs, which were increases compared to the unfilled matrix of $4.4 \%$ and $28 \%$, respectively. The nanocomposites showed toughening mechanisms imparted by both dispersed MWCNT and their agglomerates. For nanocomposites with AR-MWCNT, nanotube debonding predominated and the larger agglomerates found in this system, typically $>100 \mu \mathrm{m}$ contained weakly-bonded nanotubes, which acted as stress concentrators and reduced fracture resistance. For nanocomposites with functionalised MWCNT, the mechanisms observed were nanotube fracture and pull-out and the smaller agglomerates, containing more strongly bonded nanotubes provided toughening via crack-deflection and pinning.

\section{ACKNOWLEDGEMENTS}

Huntsman Advanced Materials and Dow Chemicals are thanked for their supply of the epoxy resin system components. The financial support of YL by the China Scholarship Council is also gratefully acknowledged. 


\section{REFERENCES}

[1] Kim M, Park Y-B, Okoli OI, Zhang C. Processing, characterization, and modeling of carbon nanotubereinforced multiscale composites. Composites Science and Technology. 2009;69:335-42.

[2] Song YS, Youn JR. Influence of dispersion states of carbon nanotubes on physical properties of epoxy nanocomposites. Carbon. 2005;43:1378-85.

[3] Ma PC, Kim J-K, Tang BZ. Effects of silane functionalization on the properties of carbon nanotube/epoxy nanocomposites. Composites Science and Technology. 2007;67(14):2965-72.

[4] Geng Y, Liu MY, Li J, Shi XM, Kim JK. Effects of surfactant treatment on mechanical and electrical properties of CNT/epoxy nanocomposites. Composites Part A: Applied Science and Manufacturing. 2008;39(12):1876-83.

[5] Shen J, Huang W, Wu L, Hu Y, Ye M. The reinforcement role of different amino-functionalized multiwalled carbon nanotubes in epoxy nanocomposites. Composites Science and Technology. 2007;67(1516):3041-50.

[6] Lachman N, Wagner HD. Correlation between interfacial molecular structure and mechanics in CNT/epoxy nano-composites. Composites: Part A. 2010;41:1093-8.

[7] Sandlera J, Shaffer MSP, Prasse T. Development of a dispersion process for carbon nanotubes in an epoxy matrix and the resulting electrical properties. Polymer. 1999;40:5967-71.

[8] Gojny FH, Wichmann MHG, Kopke U. Carbon nanotube-reinforced epoxy-composites: enhanced stiffness and fracture toughness at low nanotube content. Composites Science and Technology. 2004;64:2363-71.

[9] Yuan L, Wu D, Zhang M, Zhou W, Lin D. Rheological Percolation Behavior and Isothermal Crystallization of Poly(butyene Succinte)/Carbon Nanotube Composites. Industrial \& Engineering Chemistry Research. 2011;50(24):14186-92.

[10] Fan ZH, Advani SG. Rheology of multiwall carbon nanotube suspensions. Journal of Rheology. 2007;51(4).

[11] Bauhofer W, Kovacs JZ. A review and analysis of electrical percolation in carbon nanotube polymer composites. Composites Science and Technology. 2009;69(10):1486-98.

[12] Sumfleth J, Buschhorn S, Schulte K. Comparison of rheological and electrical percolation phenomena in carbon black and carbon nanotube filled epoxy polymers. J Mater Sci. 2011;46(3):659-69.

[13] Chapartegui M, Markaide N, Florez S, Elizetxea C, Fernandez M, Santamaría A. Specific rheological and electrical features of carbon nanotube dispersions in an epoxy matrix. Composites Science and Technology. 2010;70(5):879-84.

[14] Kim JA, Seong DG, Kang TJ, Youn JR. Effects of surface modification on rheological and mechanical properties of CNT/epoxy composites. Carbon. 2006;44(10):1898-905.

[15] Mirjalili V, Hubert P. Effect of carbon nanotube dispersion on the fracture toughness of polymers. ICCM. Edinburgh UK2009.

[16] Liu Y. Nano-reinforced Epoxy Resin for Carbon Fibre Fabric Composites. Manchester: The University of Manchester; 2016.

[17] Verdejo R, Lamoriniere S, Cottam B, Bismarck A, Shaffer M. Removal of oxidation debris from multiwalled carbon nanotubes. Chem Commun. 2007:513-5.

[18] Heister E, Lamprecht C, Neves V, Tîlmaciu C, Datas L, Flahaut E. Higher Dispersion Efficacy of Functionalized Carbon Nanotubes in Chemical and Biological Environments. ACS Nano. 2010;4(5):2615-26. 
[19] Stéfani D, Paula AJ, Vaz BG, Silva RA, Andrade NF, Justo GZ, et al. Structural and proactive safety aspects of oxidation debris from multiwalled carbon nanotubes. Journal of Hazardous Materials. 2011;189(12):391-6.

[20] Heo G-Y, Park S-J. Rheological and thermal properties of epoxy nanocomposites reinforced with alkylated multi-walled carbon nanotubes. Polymer International. 2012;61(9):1371-5.

[21] Zhu J, Wei S, Yadav A, Guo Z. Rheological behaviors and electrical conductivity of epoxy resin nanocomposites suspended with in-situ stabilized carbon nanofibers. Polymer. 2010;51(12):2643-51.

[22] Ma P-C, Siddiqui NA, Marom G, Kim J-K. Dispersion and functionalization of carbon nanotubes for polymer-based nanocomposites: A review. Composites Part A: Applied Science and Manufacturing. 2010;41(10):1345-67.

[23] Kotsilkova R. Processing-structure-properties relationships of mechanically and thermally enhanced smectite/epoxy nanocomposites. Journal of Applied Polymer Science. 2005;97(6):2499-510.

[24] Kotsilkova R, Nesheva D, Nedkov I, Krusteva E, Stavrev S. Rheological, electrical, and microwave properties of polymers with nanosized carbon particles. Journal of Applied Polymer Science. 2004;92(4):2220-7. [25] Penu C, Hu G-H, Fernandez A, Marchal P, Choplin L. Rheological and electrical percolation thresholds of carbon nanotube/polymer nanocomposites. Polymer Engineering \& Science. 2012;52(10):2173-81.

[26] Buscall R, Mills PDA. Scaling Behaviour of the Rheology of Aggregate Networks formed from Colloidal Particles. J Chem Soc, Faraday Trans I.84(12):4249-60.

[27] Kirkpatrick S. Percolation and Conduction. Reviews of Modern Physics. 1973;45(4):574-88.

[28] Wooster TJ, Abrol S, MacFarlane DR. Rheological and mechanical properties of percolated cyanate ester nanocomposites. Polymer. 2005;46(19):8011-7.

[29] Leonard F, Stein J, Wilkinson A, Withers P. 3D Characterisation of Void Distribution in Resin Film Infused Composites. Conference on Industrial Computed Tomography (ICT 2012). Wels, Austria2012.

[30] Ma P-C, Mo S-Y, Tang B-Z, Kim J-K. Dispersion, interfacial interaction and re-agglomeration of functionalized carbon nanotubes in epoxy composites. Carbon. 2010;48(6):1824-34.

[31] Behnam A, Yadienka M-R, Lolei K, Mostafa Y, Christopher TK, Pascal H, et al. Influence of the reaction stoichiometry on the mechanical and thermal properties of SWCNT-modified epoxy composites. Nanotechnology. 2013;24(26):265701.

[32] Poynton G. Multi - component Epoxy Resin Formulation for High Temperature Applications: The University of Manchester; 2014.

[33] Thostenson ET, Chou T-W. Processing-structure-multi-functional property relationship in carbon nanotube/epoxy composites. Carbon. 2006;44(14):3022-9.

[34] Gojny FH, Wichmann MHG, Fiedler B, Schulte K. Influence of different carbon nanotubes on the mechanical properties of epoxy matrix composites - A comparative study. Composites Science and Technology. 2005;65(15-16):2300-13.

[35] Kim BC, Park SW, Lee DG. Fracture toughness of the nano-particle reinforced epoxy composite. Composite Structures. 2008;86(1-3):69-77.

[36] Shin S, Jang J. The effect of amine/epoxy ratio on the fracture toughness of tetrafunctional epoxy resin. Polymer Bulletin. 1997;39(3):353-9.

[37] Mirjalili V. Aspects of the Fracture Toughness of Carbon Nanotube Modified Epoxy Polymer Composites: McGill University; 2011. 
[38] Martinez-Rubi Y, Gonzalez-Dominguez JM, Ansón-Casaos A, Kingston CT, Daroszewska M, Barnes M, et al. Tailored SWCNT functionalization optimized for compatibility with epoxy matrices. Nanotechnology. 2012;23(28):285701.

[39] Gojny FH, Nastalczyk J, Roslaniec Z. Surface modified multi-walled carbon nanotubes in CNT/epoxycomposites. Chemical Physics Letters. 2003;370(5-6):820-4.

[40] Hsieh TH, Kinloch AJ, Taylor AC, Kinloch IA. The effect of carbon nanotubes on the fracture toughness and fatigue performance of a thermosetting epoxy polymer. J Mater Sci. 2011;46(23):7525-35.

[41] Yamini S, Young RJ. Crack propagation in and fractography of epoxy resins. J Mater Sci. 1979;14(7):1609-18.

[42] Kinloch A, Young R. Fracture behaviour of polymers. London: Applied Science Publishers; 1983.

[43] Varley R, Hodgkin J, Simon G. Toughening of Trifunctional Epoxy System. V. Structure-Property Relationships of Neat Resin. Journal of Applied Polymer Science. 2000(77:):237-48. 